\title{
Primary Tree Shrew Dermis Fibroblasts Support Lytic Replication of Human Cytomegalovirus
}

\author{
Shu-Wei Dong ${ }^{1, \#}$, Ling-Shuai Jiaoo, ${ }^{1, \#}$, Ming Yang ${ }^{1, \#}$, Ying-Liang Duan ${ }^{2}$, Yi-Bo Chen ${ }^{1}$, Fei Zhao ${ }^{3}$, \\ A-Mei Zhang1, Li Liu', Min-Hua Luo ${ }^{3,}$, Xue-Shan Xia ${ }^{1},{ }^{*}$ \\ 1 Faculty of Life Science and Technology, Kunming University of Science and Technology, Kunming 650500, \\ China; dongsw@kmust.edu.cn (S.W.D.); jiaolingshuai@163.com (L.S.J.); qjyangming@163.com (M.Y.); \\ 546827735@qq.com (Y.B.C,); zam1980@kmust.edu.cn (A.M.Z); liuli2272@163.com (L.L.) \\ 2 The Key Laboratory of Tropical and Subtropical Animal Viral Diseases in Yunnan province, Kunming \\ 650224, China; water.d@qq.com (Y.L.D.) \\ 3 State Key Laboratory of Virology, Wuhan Institute of Virology, Chinese Academy of Sciences, Wuhan \\ 430071, China; zhaofei@wh.iov.cn (F.Z.) \\ * Correspondence: luomh@wh.iov.cn; Tel: 86-027-8719-7600; xueshanxia@kmust.edu.cn; Tel: 86-0871- \\ 6592-0756 \\ \# These authors contributed equally as co-first authors of this work.
}

\begin{abstract}
As a universal pathogen leading to neonatal defects and transplant failure, Human cytomegalovirus (HCMV) has strict species specificity that the inability to using this virus in animals has hampered its pathogenesis study. However, the mechanism of cross-species barrier remains elusive that no non-human cell model has been established to fill this knowledge gap. We observed that primary dermis fibroblasts (TSDF) isolated from the Chinese tree shrew (Tupaia belangeri chinensis), a small laboratory animal with close affinity to primates, were permissive to HCMV replication. In TSDF infected with GFP-expressing HCMV, the green fluorescence and cytopathic effect were observed and the expression of 3 kinetic genes and replication of viral genome were detected. The cell-free viruses produced in TSDF reached $10^{3} \mathrm{pfu} / \mathrm{mL}$ at $96 \mathrm{hpi}$, which were 10-fold lower than in primary human foreskin fibroblasts. Our results demonstrated that TSDF supported low level of lytic replication of HCMV. The TSDF model provides a useful platform for the mechanism study of species barrier of HCMV.
\end{abstract}

Keywords: Human cytomegalovirus; primary tree shrew dermis fibroblasts; cross-species infection; lytic replication.

\section{Introduction}

Human cytomegalovirus (HCMV) is a worldwide opportunistic pathogen and the leading infectious cause of severe diseases in immunodeficiency individuals. Its pathogenesis involves lytic replication in cells, during which the expression of viral genes is temporally controlled $[1,2]$. Immediate early (IE) genes (such as IE1) are expressed after viral genome delivered into the nucleus, then the expression of early genes (such as UL44) is activated by IE proteins which takes part in viral genome replication. Late gene expression (such as pp28) depends on E genes expression and controls envelopment and egress of progeny viruses. There are various types of human cells supporting the expression of the 3 kinetics proteins and production of viral particles. However, infection of cells from commonly used laboratory animals was abortive, which is the result of the strict species of HCMV [3-6]. The inability to use HCMV in animal has hampered its pathogenesis study. In order to overcome this limitation, it is important to investigate the molecular mechanism of the species specificity, for which the establishment of a cross-species infection model is indispensable.

In the past decades, both in vitro and in vivo cross-species infection of HCMV have been tried by several groups. Rodents are the most popular laboratory animals that the humanized-mouse engrafted with human tissues is up till now the only animal model for direct in vivo study of 
HCMV, but viral infection limited in implanted cells with human origin [7]. On the other hand, there were two reports presenting low level of HCMV replication in non-human cells, which were primary chimpanzee skin fibroblasts $(\mathrm{CF})$ and porcine endothelial cells, respectively $[8,9]$. Unfortunately, chimpanzees are forbidden to be used in invasive researches due to ethical concern and their endangered state [10], while the viral growth kinetic was not determined in porcine endothelial cells. Despite the limitations of these studies, we are enlightened that at least two aspects would benefit the establishment of cross-species model for HCMV infection. First, the species specificity is stricter in vivo that a non-human cell model for HCMV infection would be easier to be established. Second, an animal with high similarity to human is more likely to support productive infection of HCMV, in consideration of the high level of adaptation of this virus to human.

In recent years, the Chinese tree shrew (Tupaia belangeri chinensis) was found to be genetically close to primates and its genes or pathways playing a role in infectious diseases have higher identity to human [11,12]. Meanwhile, it has economical maintenance costs and a small body size. These advantages enable its application in pathological research of many human viruses including Herpes simplex virus type $1[12,13]$. For HCMV, these advantages suggest a potential susceptibility of the Chinese tree shrew to this viral infection. Since the species specificity is less stringent in vitro infection and infection characteristic would be closer to real state in primary cells, we investigated the susceptibility of primary tree shrew dermis fibroblasts (TSDF) to HCMV infection.

Here, TSDF was established in culture and a bacterial artificial chromosome (BAC) clone of HCMV Towne strain which expresses a $g f p$ gene in the viral genome was used to monitor lytic replication [14]. The full cycle of viral replication was achieved in TSDF by observing cytopathic effect (CPE) and GFP expression, detecting the expressions of IE1, UL44, and pp28 and the genome replication. By plaque forming assay in monolayer of primary human foreskin fibroblasts (HFF), cell-free viruses produced in TSDF led to the expression of green fluorescence, and the highest titer reached $10^{3} \mathrm{PFU} / \mathrm{mL}$ at $96 \mathrm{hpi}$, which was 10 -fold lower than that in HFF. These results confirmed low level of productive replication of HCMV in TSDF. The TSDF model provides a useful platform for study of the molecular mechanism of species specificity of HCMV.

\section{Materials and Methods}

\subsection{Cells and cell culture}

The 3 months old first filial-generation Chinese tree shrews used for primary cells isolation were purchased from the Institute of Medical Biology, Chinese Academy of Medical Sciences. The primary TSDF was obtained by enzyme digestion method with $0.1 \%$ type II collagenase [15]. Both TSDF and HFF were cultured in minimal essential medium (MEM) medium (Hyclone) and supplemented with $10 \%$ fetal bovine serum (FBS), penicillin and streptomycin (100 U/ml and 100 $\mathrm{g} / \mathrm{ml}$, respectively, Gibco). Low serum growth supplement (Gibco) was added for TSDF. Cell cultural condition was maintained at $37^{\circ} \mathrm{C}$ in a humidified atmosphere containing $5 \% \mathrm{CO}_{2}$. The morphology of cells was observed with Leica Microsystems (Leica).

2.2 Virus and viral infection

A BAC clone of HCMV Towne strain expressing a $g f p$ gene in the viral genome was propagated in HFF with low MOI (0.02) and titrated by plaque assay on monolayer of HFF as described previously [16]. Cells were infected with HCMV at an MOI of 1 or 0.5 and harvest at indicated times for observation of $\mathrm{CPE}$ and detection of viral replication. The rate of GFP-positive cells was calculated by counting 1,000 cells from 3 to 4 fields.

\subsection{DNA isolation and quantitative PCR assay}

DNA was isolated from cells with TIANamp Genomic DNA Kit (TIANGEN). HCMV fragment was amplified with $50 \mathrm{ng}$ DNA and primers specific to ul83 (Forword -5'GCGAGACCGTGGAACTGC 3', Reverse -5' TTGCCCTGGATGCGATACTG 3') by using ChamQ SYBR q-PCR Master Mix Kit (Vazyme) and sequenced. The qPCR was performed as follow: 1 cycle of $5 \mathrm{~min}$ at $95^{\circ} \mathrm{C} ; 40$ cycles of $10 \mathrm{sec}$ at $95^{\circ} \mathrm{C}$ and $34 \mathrm{sec}$ at $60^{\circ} \mathrm{C} ; 1 \mathrm{cycle}$ of $15 \mathrm{sec}$ at $95^{\circ} \mathrm{C}, 60 \mathrm{sec}$ at $60^{\circ} \mathrm{C}$, and $15 \mathrm{sec}$ at $95^{\circ} \mathrm{C}$. The plasmid pcDNA3.0-UL83 was used to get a standard curve and the copy number of HCMV in different cells was subsequently calculated. 


\subsection{Western Blot}

Cells were collected and washed with PBS (Gibco). After centrifuge at 12,000 rpm, samples were added with $100 \mu \mathrm{L}$ RIPA and incubated on ice for $1 \mathrm{~h}$ for total protein preparation. Twenty microliter of total protein quantified with BCA Protein Assay Kit (TaKaRa) was used for protein separation with SDS-PAGE (Bio-Rad). The protein was transferred to a polyvinylidene fluoride membrane (Milipore) and the membrane was blocked and incubated with primary and secondary antibodies. Mouse monoclonal antibodies against IE1 (Thermo Fisher), UL44 (Abcam), and pp28 (Abcam) were 1:1000 diluted, and mouse monoclonal antibodies against $\beta$-actin were 1:5000 diluted (Abcam). Horseradish peroxidase (HRP)-goat anti-mouse IgG (Abcam) was 1:5000 diluted. The membranes were treated with enhanced chemiluminescence reagents (GE) and analyzed by using Imaging system (Bio-Rad).

2.5 Immunofluorescence assay (IFA)

Cells were cultured in 10-mm dishes containing coverslips and at least 3 coverslips were used for each sample. At indicated times, the coverslips were rinsed with PBS, fixed with $4 \%$ formaldehyde, permeabilized with $1 \%$ Triton-100, blocked with corresponding antibodies, and counterstained with $1 \mu \mathrm{g} / \mathrm{ml}$ DAPI (Roche). For detection of vimentin expression, TSDF-containing coverslips were first incubated with rabbit monoclonal antibodies against human vimentin (1:1000 diluted, Abcam) and mouse monoclonal antibodies against $\beta$-actin (1:1000 diluted, Abcam) for $2 \mathrm{~h}$, then with FITC-conjugated goat polyclonal antibodies against rabbit IgG (1:500 diluted, Abcam) and rhodamine-conjugated goat polyclonal antibodies against mouse IgG (1:200, Thermo Fisher). The detection of cytokeratin 10 was carried out in the same way except rabbit monoclonal antibodies against cytokeratin 10 (1:200 diluted, Abcam) being used. The cells were visualized under Leica DMI3000B Manual Inverted Microscope and image quantification was carried out using NIS-Elements F 4.00.00 software (Leica).

2.6 Ethics statement and statistical statement

All experiments and protocols conducted in this study were reviewed and approved by the Institutional Ethical Committee of Kunming University of Science and Technology. Statistical analyses were performed with GraphPad Prism 5 (GraphPad Software), using Student's t-test or Analysis of Variance (ANOVA) test. The average results were shown as the mean \pm standard deviation (SD). Significance was considered when $\mathrm{p}<0.05$.

\section{Results}

\subsection{Isolation and characterization of primary TSDF}

In consideration of reported permissiveness of primary skin fibroblasts from chimpanzee for HCMV infection and close relationship to primates, primary TSDF were isolated. After mechanical and enzymatic dissociation, the cells attached and spread on the plate, and gradually became confluent. Most cells were with a uniform spindle shape and parallel arranged when they grew densely, the morphology of which was similar to fibroblasts (Fig.1A). The cell type was further determined by detection of the expression of vimentin and cytokeratin 10 (Fig.1B), the marker for fibroblasts and epithelial cells, respectively [15-17]. The vimentin expression was observed in most TSDF (green fluorescence) and co-localized with $\beta$-actin (red fluorescence) in cytoplasm, which was consistent with characteristic of fibroblasts. However, the expression of cytokeratin 10 was not detected suggesting that no epithelial cells or the expression level was below the detection limit. Therefore, primary TSDF in culture was established. 


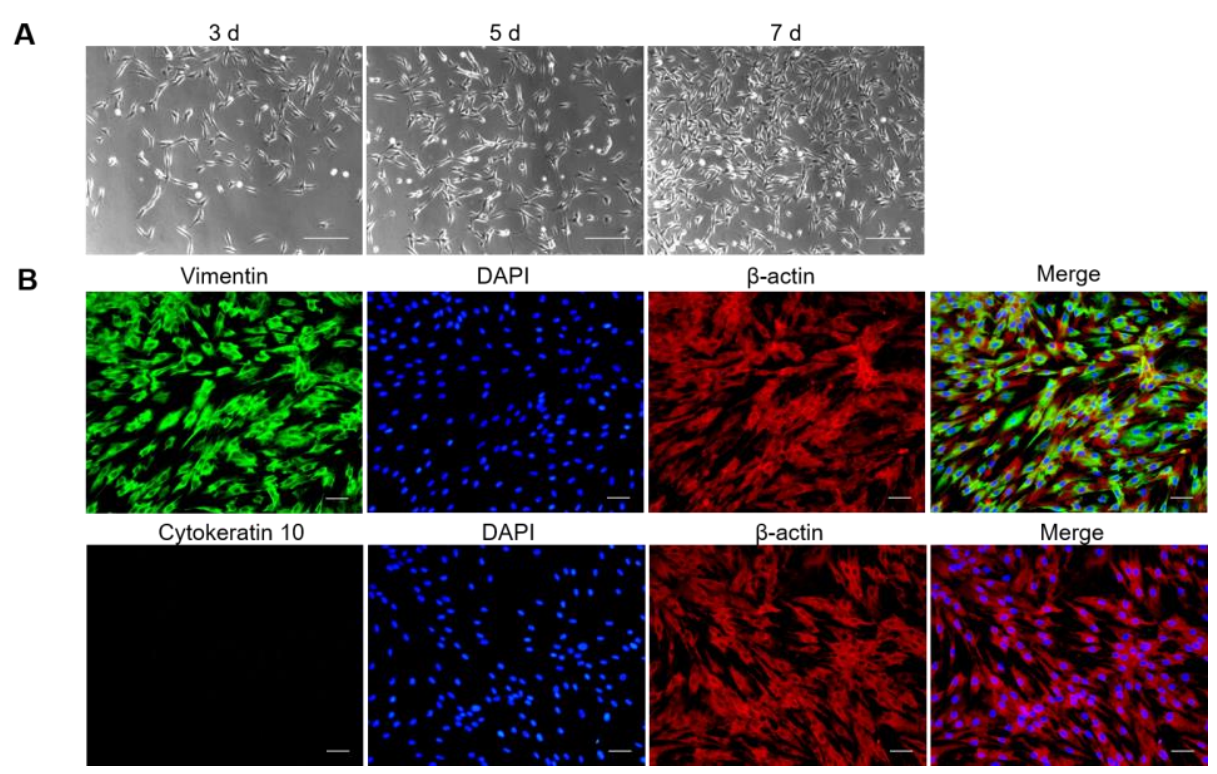

Figure 1. Isolation and characterization of TSDF. (A) The morphology of TSDF cultured for 3, 5 and 7 days, respectively. (B) Vimentin and cytokeratin 10 expressions in TSDF. The green fluorescence showed vimentin and cytokeratin 10, $\beta$-actin marked cytoplasm (red fluorescence), and DAPI marked nuclear (blue fluorescence). Bar= $100 \mu \mathrm{m}$.

\subsection{HCMV infection induced GFP expression and CPE in TSDF}

To initially detect the susceptibility to HCMV infection, primary TSDF was infected at an MOI of 1 with GFP-expressing HCMV which is convenient for monitoring lytic replication [14,18]. Both GFP expression and CPE were observed at 24, 36, 48, 72, 96 hpi (Fig.2), and HFF were used as positive control. In HFF, weak expression of green fluorescence was detected at $24 \mathrm{hpi}$ and more than half cells were GFP positive from $36 \mathrm{hpi}$. The GFP-positive HFF was close to 100\% at $96 \mathrm{hpi}$. In TSDF, GFP expression was delayed that weak green fluorescence appeared at $36 \mathrm{hpi}$. The maximum rate of GFP-positive TSDF was $70 \%$ at $72 \mathrm{hpi}$ then decreased to $50 \%$ at $96 \mathrm{hpi}$. The overall GFP intensity was much stronger in HFF than in TSDF. Meanwhile, the typical cell rounding and enlargement were presented in HCMV-infected HFF. For TSDF, not only were cell rounding and enlargement observed, but also cell detachment occurred at late infection period. However, in mock-infected cells, no visible CPE was observed. The CPE and expression of green fluorescence implied a possibility of lytic replication in HCMV-infected TSDF. 

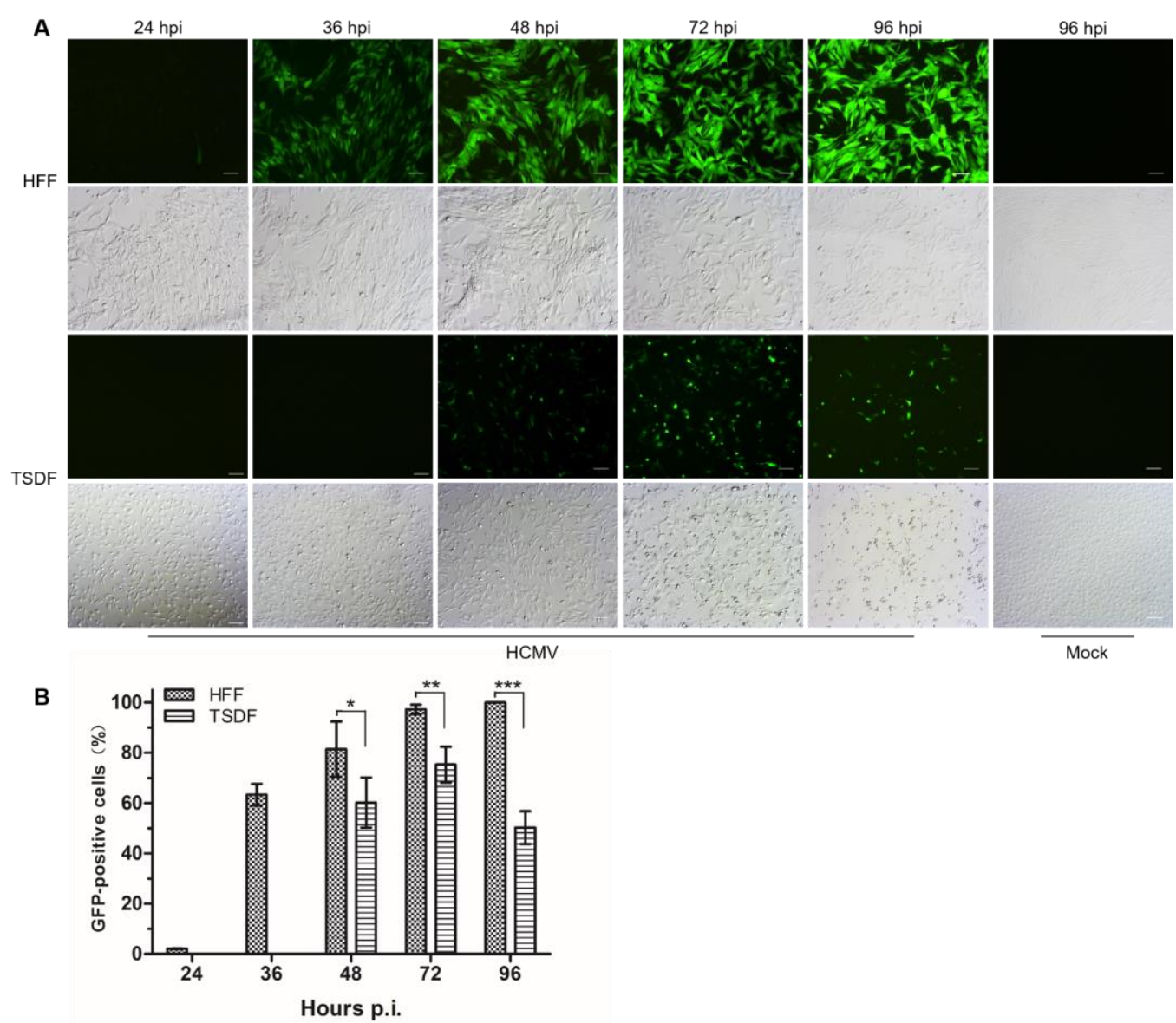

Figure 2. HCMV infection induced GFP expression and CPE in TSDF. (A) The expression of GFP and CPE were observed in HCMV-infected HFF and TSDF at 24, 36, 48, 72, and 96 hpi. Mock-infected cells at 96 hpi were shown as negative controls. Bar=100 $\mu \mathrm{m}$. (B) The percentage of GFP-positive cells was calculated respectively for HFF and TSDF. ${ }^{*}$ means $p<0.05,{ }^{* *}$ means $p<0.01$, ${ }^{* * *}$ means $\mathrm{p}<0.001$.

\subsection{TSDF supported HCMV gene expression and genome replication}

Previous studies showed that HCMV cross-infection failure was due to a blockage at post-entry stage that early gene expression and DNA replication could not be achieved [5,6,19]. In order to determine whether TSDF supported authentic replication of this virus, TSDF and HFF were infected at an MOI of 0.5 and the expressions of IE1, UL44, pp28, and $\beta$-actin were detected by western blot and genome copy number was analyzed by quantitative PCR (qPCR). As shown in Fig.3A, these 3 viral genes expressed in HFF and the expression levels increased over time. In TSDF, the expressions of IE1 and UL44 were increased from $24 \mathrm{hpi}$, and the expression of pp28 was detected from $72 \mathrm{hpi}$ to 120 hpi. The genome replication level was quantified with UL83 primers (Fig.3B). In HFF, the viral DNA copy number was from $10^{4}$ and $10^{7}$ per 50 ng total DNA from 24 to 168 hpi. However, the viral DNA copy number kept at $10^{3}$ per $50 \mathrm{ng}$ total DNA in TSDF since $24 \mathrm{hpi}$. The PCR fragments were sequenced and confirmed as HCMV. The low replication level was in accordance with the weak expression of pp28. These results demonstrated that TSDF supported the 3 kinetic genes expressions and genome replication of HCMV. 
A

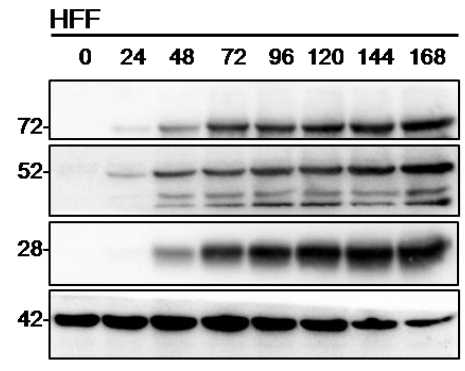

TSDF

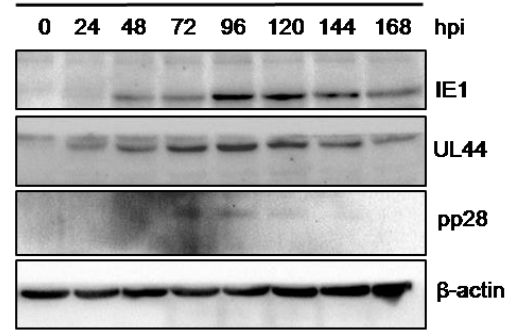

B

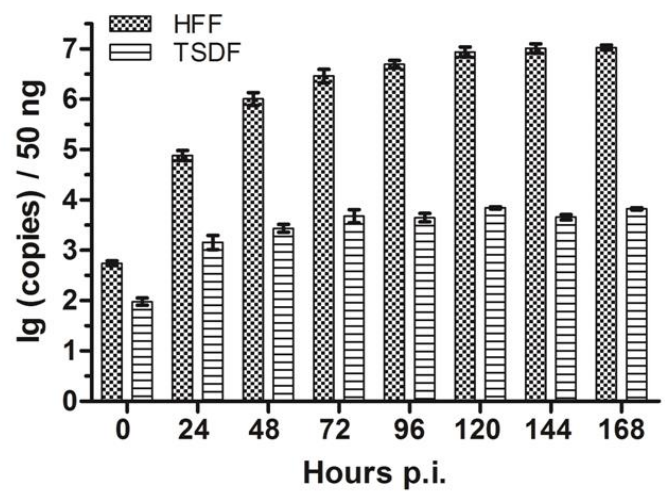

Figure 3. TSDF supported the 3 kinetics genes expressions and genome replication of HCMV. (A) The expression of IE1, UL44, pp28, and $\beta$-actin was detected by western blot at specific infection period. (B) The genome replication of HCMV was analyzed by qPCR with UL83 primers.

\subsection{TSDF supports production of Infectious progeny virus which can infect HFF}

We next sought to ask whether infectious progeny viruses could be produced in TSDF besides late protein expression and viral genome replication. The growth curve of HCMV in TSDF was determined by plaque forming assay on fresh HFF monolayer and compared with virus yield in HFF (Fig. 4). The GFP expression and small plaques were detected in HFF inoculated with cell-free viruses produced in TSDF (Fig.4B). From 24 to $48 \mathrm{hpi}$, the titer in both TSDF and HFF decreased to similar amount, but it began to increase from $72 \mathrm{hpi}$. The amount of cell-free viruses reached the highest level in TSDF at $96 \mathrm{hpi}$, which was $10^{3} \mathrm{PFU} / \mathrm{mL}$ and 10-fold lower than that in HFF. After 96 hpi, the titer in TSDF gradually decreased while it kept growing in HFF and reached $10^{5} \mathrm{PFU} / \mathrm{mL}$ at 168 hpi. This decrease of amount of cell-free viruses was consistent with cell detachment and decrease of pp28 expression level, which suggested the viral replication was somehow disrupted in late infection period. In order to further confirm that progeny cell-free viruses were actually produced in TSDF, an inactivation control was conducted that the same amount of viruses remaining in the supernatant at $24 \mathrm{hpi}$ was inoculated to fresh medium and kept at the same culture condition. Interestingly, the titer in inactivation control kept decreasing after $48 \mathrm{hpi}$ and it was significantly lower than in TSDF at 72, 96, 120, 144, and $168 \mathrm{hpi} \mathrm{(p<0.05).} \mathrm{At} 168 \mathrm{hpi}$, there were almost no infectious viruses in inactivation control but there were still $5.59 \pm 0.58 \times 10 \mathrm{PFU} / \mathrm{mL}$ of cell-free viruses in TSDF. Based on the requirement of 48 to 72 hours to complete one replication cycle of HCMV, the growth curve showed the achievement of at least one cycle of viral replication in TSDF. Taken together, our results confirmed productive replication of HCMV in TSDF. 
A

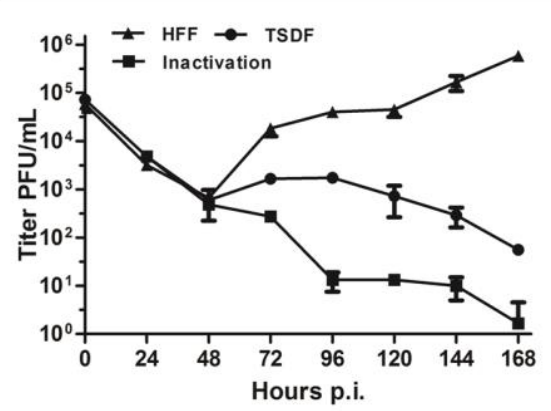

B

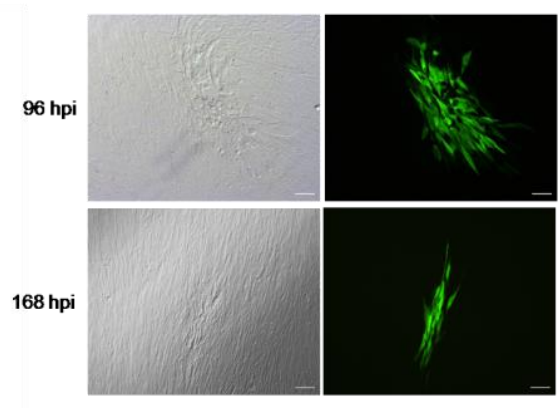

Figure 4. TSDF supports production of infectious progeny virus which can infect HFF. (A) Growth kinetic of HCMV on HFF and TSDF was determined. An inactivation control was carried out by keeping the same amount of viruses remained in supernatant of TSDF at $24 \mathrm{hpi}$ in fresh medium under the same cultural condition. (B) The formation of plaques and expression of GFP were observed in HFF infected with cell-free viruses produced in TSDF. Bar=100 $\mu \mathrm{m}$.

\section{Discussion}

As a universal pathogen leading to neonatal defects and transplant failure, the inability to applying HCMV in animal model due to the cross-species barrier of this viral infection, has impeded its pathogenesis study. In order to overcome this limitation, a non-human cell model supporting lytic replication of this virus is critical for the mechanism study of its strict species specificity. Previous reports implied that an animal with high similarity to human has a potential to be infected by HCMV [8], and the Chinese tree shrew was proved to be genetically close to primates and useful for pathological research of several human viruses [12]. Herein, we investigated the susceptibility of primary TSDF to HCMV infection. By identification of CPE, GFP expression, lytic gene expression, viral genome replication, and viral growth kinetics, we showed that HCMV could productively infect TSDF.

It is important to choose proper cell types and strains to establish a cell model for cross-species infection of HCMV. In human, primary fibroblasts with skin or lung origin are mostly used to study in vitro HCMV infection because it is one of the main target cell types, easy to culture, permissive for all strains, and supports production of high titer of cell-free virus [20]. Towne strain, one of the mostly used laboratory strain, has been reported to be permissive for HCMV infection of CF. In this study, TSDF was successfully established in culture by examination of morphology and positive expression of fibroblast marker protein. The infection was conducted with a Towne strain variant expressing a gfp gene in the viral genome which has been proved to be convenient to monitor viral replication [18]. In HCMV-infected TSDF, by detecting expression of the green fluorescence and 3 kinetic proteins and production of cell-free virus, it demonstrated successful cross-species infection in this condition. Meanwhile, compared to TSDF, we indeed observed different susceptibility of primary fibroblasts from lung (TSLF) and epithelial cells from kidney (TSKE) to HCMV. The IE-1 expression was less efficient in TSLF than in TSDF and no expression could be detected in TSKE (data not shown). So far, TSDF is easy to be cultured and supports complete replication cycle of HCMV that this cell model would be useful for study cross-species infection of this virus.

The species specificity of HCMV is the result of coevolution with human host that the interplay between the virus and the Chinese tree shrew would affect its cross-species infection. Although the mechanism of cross-species barrier of HCMV is still unknown, studies on other CMVs have implied that multiple factors play a part in this process [21-26]. On the viral side, UL128 and UL130 which are the subunit of pentameric complex in the viral envelope helped rhesus macaques CMV (RhCMV) infect cynomolgus macaques both in vitro and in vivo [26]. Since complete pentameric complex is necessary for infection of epithelial and endothelial cells, it suggested that cross-species infection would benefit from broadening the cell types a virus entering. There are various HCMV strains which could be classified as laboratory strains, such as Towne, and clinical strains. The laboratory 
strains was highly adapted to fibroblasts and could produce high titer of cell-free viruses, while clinical strains have extended tropism and could infected epithelial and endothelial cells besides fibroblasts [27]. Whether clinical strains of HCMV would productively infected TSDF should be investigated in future. On the other side, host factors involved in anti-viral response, including apoptosis and ND10 complex, were also reported to influence cross-species infection of CMVs $[21,22,26]$. In this study, we observed cell detachment in HCMV-infected TSDF at late infection period. It is unclear what factors caused cell death and whether it has effects on virus multiplication. By using TSDF model, examination of factors that improve production of virus particle and relieve cell death would provide perspectives on mechanism of species specificity of HCMV.

In conclusion, the data of the present study showed productive infection of TSDF with HCMV. This TSDF model provides a convenient platform for investigation of key factors influencing cross-species multiplication of HCMV. Meanwhile, the characteristics of this viral infection of other types of cells from the Chinese tree shrew should be further studied to explore the utility of this animal for HCMV infection.

Author Contributions: S.W.D conceived the experiments. S.W.D. and L.S.J designed the experimental flow. L.S.J., M.Y., Y.L.D., and Y.B.C. performed the experiments. L.S.J. performed statistical analyses. S.W.D. wrote the manuscript. F.Z., A.M.Z., and L.L. reviewed the manuscript. M.H.L. and X.S.X. supervised the project.

Funding: This research was supported by National Science and Key Technology Support Program (2014BAI01B01), Applied Basic Research Projects of Yunnan Province (2018FD034), and Yunnan Provincial Department of Education Science Research Fund Project (1405189906).

Acknowledgments: We thank Dr. Joseph Sriyal Malik Peiris for critical reading of the manuscript.

Conflicts of Interest: The authors declare no conflict of interest.

\section{References}

1. Wathen, M.W.; Thomsen, D.R.; Stinski, M.F. Temporal regulation of human cytomegalovirus transcription at immediate early and early times after infection. J. Virol. 1981, 38, 446-459.

2. Wathen, M.W.; Stinski, M.F. Temporal patterns of human cytomegalovirus transcription: mapping the viral RNAs synthesized at immediate early, early, and late times after infection. J. Virol. 1982, 41, 462-477.

3. Kim, K.S.; Carp, R.I. Abortive infection of human diploid cells by murine cytomegalovirus. Infect. Immun. 1972, 6, 793-797.

4. Fioretti, A.; Furukawa, T.; Santoli, D.; Plotkin, S.A. Nonproductive infection of guinea pig cells with human cytomegalovirus. J. Virol. 1973, 11, 998-1003.

5. Lafemina, R.L.; Hayward, G.S. Differences in cell-type-specific blocks to immediate early gene expression and DNA replication of human, simian and murine cytomegalovirus. J. Gen. Virol. 1988, 69 ( Pt 2), 355-374, doi:10.1099/0022-1317-69-2-355.

6. Garcia-Ramirez, J.J.; Ruchti, F.; Huang, H.; Simmen, K.; Angulo, A.; Ghazal, P. Dominance of virus over host factors in cross-species activation of human cytomegalovirus early gene expression. J. Virol. 2001, 75, 26-35, doi:10.1128/jvi.75.1.26-35.2001.

7. Crawford, L.B.; Streblow, D.N.; Hakki, M.; Nelson, J.A.; Caposio, P. Humanized mouse models of human cytomegalovirus infection. Curr Opin Virol 2015, 13, 86-92, doi:10.1016/j.coviro.2015.06.006.

8. Perot, K.; Walker, C.M.; Spaete, R.R. Primary chimpanzee skin fibroblast cells are fully permissive for human cytomegalovirus replication. J. Gen. Virol. 1992, 73 ( Pt 12), 3281-3284, doi:10.1099/0022-1317-73-12-3281. 
9. Millard, A.L.; Haberli, L.; Sinzger, C.; Ghielmetti, M.; Schneider, M.K.; Bossart, W.; Seebach, J.D.; Mueller, N.J. Efficiency of porcine endothelial cell infection with human cytomegalovirus depends on both virus tropism and endothelial cell vascular origin. Xenotransplantation 2010, 17, 274-287, doi:10.1111/j.1399-3089.2010.00594.x.

10. Bennett, A.J.; Panicker, S. Broader impacts: international implications and integrative ethical consideration of policy decisions about US chimpanzee research. Am. J. Primatol. 2016, 78, 1282-1303, doi:10.1002/ajp.22582.

11. Fan, Y.; Huang, Z.Y.; Cao, C.C.; Chen, C.S.; Chen, Y.X.; Fan, D.D.; He, J.; Hou, H.L.; Hu, L.; Hu, X.T., et al. Genome of the Chinese tree shrew. Nat Commun 2013, 4, 1426, doi:10.1038/ncomms2416.

12. Yao, Y.G. Creating animal models, why not use the Chinese tree shrew (Tupaia belangeri chinensis)? Zool. Res. 2017, 38, 118-126, doi:10.24272/j.issn.2095-8137.2017.032.

13. Li, L.; Li, Z.; Wang, E.; Yang, R.; Xiao, Y.; Han, H.; Lang, F.; Li, X.; Xia, Y.; Gao, F., et al. Herpes Simplex Virus 1 Infection of Tree Shrews Differs from That of Mice in the Severity of Acute Infection and Viral Transcription in the Peripheral Nervous System. J. Virol. 2016, 90, 790-804, doi:10.1128/JVI.02258-15.

14. Marchini, A.; Liu, H.; Zhu, H. Human cytomegalovirus with IE-2 (UL122) deleted fails to express early lytic genes. J. Virol. 2001, 75, 1870-1878, doi:10.1128/JVI.75.4.1870-1878.2001.

15. Rittie, L.; Fisher, G.J. Isolation and culture of skin fibroblasts. Methods Mol Med 2005, 117, 83-98, doi:10.1385/1-59259-940-0:083.

16. Tamashiro, J.C.; Hock, L.J.; Spector, D.H. Construction of a cloned library of the EcoRI fragments from the human cytomegalovirus genome (strain AD169). Journal of virology 1982, 42, 547-557.

17. Franke, W.W.; Schmid, E.; Osborn, M.; Weber, K. Different intermediate-sized filaments distinguished by immunofluorescence microscopy. Proc Natl Acad Sci U S A 1978, 75, 5034-5038.

18. Demirkesen, C.; Hoede, N.; Moll, R. Epithelial markers and differentiation in adnexal neoplasms of the skin: an immunohistochemical study including individual cytokeratins. J Cutan Pathol 1995, 22, 518-535.

19. Herrmann, H.; Haner, M.; Brettel, M.; Muller, S.A.; Goldie, K.N.; Fedtke, B.; Lustig, A.; Franke, W.W.; Aebi, U. Structure and assembly properties of the intermediate filament protein vimentin: the role of its head, rod and tail domains. J. Mol. Biol. 1996, 264, 933-953, doi:10.1006/jmbi.1996.0688.

20. Cheng, S.; Jiang, X.; Yang, B.; Wen, L.; Zhao, F.; Zeng, W.B.; Liu, X.J.; Dong, X.; Sun, J.Y.; Ming, Y.Z., et al. Infected T98G glioblastoma cells support human cytomegalovirus reactivation from latency. Virology 2017, 510, 205-215, doi:10.1016/j.virol.2017.07.023.

21. DeMarchi, J.M. Nature of the block in the expression of some early virus genes in cells abortively infected with human cytomegalovirus. Virology 1983, 129, 287-297.

22. Fortunato, E.A. Use of diploid human fibroblasts as a model system to culture, grow, and study human cytomegalovirus infection. Methods Mol Biol 2014, 1119, 47-57, doi:10.1007/978-1-62703-788-4_4.

23. Jurak, I.; Brune, W. Induction of apoptosis limits cytomegalovirus cross-species infection. EMBO J. 2006, 25, 2634-2642, doi:10.1038/sj.emboj.7601133.

24. Tang, Q.; Maul, G.G. Mouse cytomegalovirus crosses the species barrier with help from a few human cytomegalovirus proteins. J. Virol. 2006, 80, 7510-7521, doi:10.1128/JVI.00684-06.

25. Schumacher, U.; Handke, W.; Jurak, I.; Brune, W. Mutations in the M112/M113-coding region facilitate murine cytomegalovirus replication in human cells. J. Virol. 2010, 84, 7994-8006, doi:10.1128/JVI.02624-09. 
26. Marsh, A.K.; Ambagala, A.P.; Perciani, C.T.; Russell, J.N.; Chan, J.K.; Janes, M.; Antony, J.M.; Pilon, R.; Sandstrom, P.; Willer, D.O., et al. Examining the species-specificity of rhesus macaque cytomegalovirus (RhCMV) in cynomolgus macaques. PLoS One 2015, 10, e0121339, doi:10.1371/journal.pone.0121339.

27. Ostermann, E.; Pawletko, K.; Indenbirken, D.; Schumacher, U.; Brune, W. Stepwise adaptation of murine cytomegalovirus to cells of a foreign host for identification of host range determinants. Med. Microbiol. Immunol. 2015, 204, 461-469, doi:10.1007/s00430-015-0400-7.

28. Burwitz, B.J.; Malouli, D.; Bimber, B.N.; Reed, J.S.; Ventura, A.B.; Hancock, M.H.; Uebelhoer, L.S.; Bhusari, A.; Hammond, K.B.; Espinosa Trethewy, R.G., et al. Cross-Species Rhesus Cytomegalovirus Infection of Cynomolgus Macaques. PLoS Pathog 2016, 12, e1006014, doi:10.1371/journal.ppat.1006014.

29. Frascaroli, G.; Sinzger, C. Distinct properties of human cytomegalovirus strains and the appropriate choice of strains for particular studies. Methods Mol Biol 2014, 1119, 29-46, doi:10.1007/978-1-62703-788-4_3. 